\title{
Libro: Trabajo, ingresos y políticas en Argentina. Contribuciones para pensar el siglo XXI
}

Javier Lindenboim (compilador)

Eudeba, Buenos Aires, 2008. (375 pp.)

\section{Andrea Delfino}

Universidad Nacional del Litoral / Universidad Nacional de Rosario

Mail: andelfino@yahoo.com.ar

Desde la Modernidad el trabajo tuvo la función manifiesta de proveer ingresos, constituyéndose, de esta manera, no sólo en el sustento a partir del cual se derivaron las condiciones materiales de vida de la población sino también en la herramienta que permitió que se enlazaran buena parte de las garantías contra las inseguridades. De forma conjunta, el trabajo supuso la sujeción de los individuos al orden social a través de la interiorización de dispositivos disciplinares. Tal como señalara Polanyi (2006), el mercado de trabajo se constituyó en un "molino satánico" porque su institucionalización extendió el principio de la auto-regulación del mercado al trabajo vivo.

Sin embargo, desde el último cuarto del siglo XX se vienen sucediendo profundas modificaciones estructurales producto de la conjunción de cambios sustantivos en los modos de producción junto al debilitamiento y virtual extinción de las protecciones organizadas por el Estado de Bienestar. Las nociones de peligro y riesgo emergen como significantes del presente y se oponen drásticamente a las ideas de confianza y seguridad características de la época precedente.

Es justamente en este punto neurálgico - donde se entrelaza el trabajo, la política y la vida cotidiana- donde se inscribe el libro organizado por Javier Lindenboim. Con los aportes de Luis Beccaria, Guillermina Comas, Claudia Danani, Juan Graña, Estela Grassi, Pablo Gutiérrez Agritos, Damián Kennedy, Alejandro Lavopa, Roxana Maurizio, Julio C. Neffa, Diego Quartulli, Agustín Salvia, Federico Stefani y el propio Javier Lindenboim, el libro se propone abordar la problemática de las transformaciones del nuevo siglo en Argentina, focalizándose en el estudio del mercado de trabajo. En este sentido, "Trabajo, ingresos y políticas en Argentina. Contribuciones para pensar el siglo XXI" constituye una línea de continuidad con el anterior trabajo editorial organizado, en 2003, por Lindenboim y Danani, "Entre el trabajo y la política. Las reformas de las politicas sociales argentinas en perspectiva comparada".

La continuidad entre ambas obras no es sólo temporal, sino que ambas comparten un aparato conceptual común centrado en un doble cuestionamiento: a la concepción de autonomía de la lógica económica y a la noción de "retirada del Estado", como óptica de lectura de las trasformaciones en el aparato del Estado durante la hegemonía neoliberal.

En relación con la primera de las críticas, el mercado de trabajo no sólo es entendido como la institución en la que se constituyen las condiciones de venta y uso 
de la fuerza de trabajo sino también como el escenario en el cual las formas de organización de la vida social devienen en "vida" a secas, pues devienen en vida de las personas, tal como plantean Grassi y Danani. En este mercado, las políticas cumplen un papel constitutivo. Allí se articula el ámbito de la economía y del trabajo con las políticas y la política, conformando una relación que adquiere un lugar estructurante en la vida de las personas. El cuestionamiento explícito a la concepción de la economía como un dominio autónomo y autosuficiente conlleva el abordaje de las formas de intervención sobre el mercado de trabajo y, consecuentemente, de la producción de dos organizadores de la vida social e individual: el orden político-institucional y las condiciones de vida en sentido amplio. Así, el ámbito laboral es abordado como el espacio donde se configuran las condiciones de participación en la distribución de los beneficios producidos y acumulados; se conforman las condiciones de acceso al consumo y, en buena medida, también se escribe un capítulo fundamental de la producción de identidad, reconocimiento y subjetividad.

En relación con la segunda de las críticas, en la obra subyace la tesis acerca de que las expresiones "retirada del Estado" y/o "disminución o desaparición de la intervención del Estado" sustraen del debate y de la comprensión la monumental labor de construcción cotidiana de una nueva sociedad, que sólo pudo llevarse a cabo por y desde el corazón mismo del Estado. Así, la sociedad que ve la luz luego de treinta años de proyectos políticos neoliberales es una sociedad cuyo Estado no es más pequeño, ni menos "interventor", sino un Estado distinto, cuyas acciones y políticas son tan intensas como lo eran antes, pero portadoras de significados y orientaciones diferentes, o incluso opuestas en muchos casos.

Esta tesis tributaria de los más recientes debates en el campo de la antropología política puede ser encontrada también en las obras de Grimson (2003) y de Trouillot (2001). Para Grimson (2003), nominar genéricamente de "retirada" la reingeniería del Estado no resulta del todo adecuado para dar cuenta de los aspectos y los sentidos en los cuales el Estado se ha retirado, en los cuales se ha transformado y de las consecuencias e impactos que tuvo este proceso en la vida de los diferentes sectores sociales. En una línea que profundiza estas ideas, Trouillot (2001) señala que los planteos relacionados con la declinación de la relevancia del Estado son en el mejor de los casos, prematuros. Para el autor, estos postulados desconocen que los procesos no se han desarrollado de forma unilateral, sino que los cambios son múltiples y a veces también contradictorios.

La obra compilada por Lindenboim se estructura en tres secciones. Las dos primeras proporcionan una mirada más propiamente estructural de los procesos, mientras que la tercera se introduce de forma directa en diferentes formas de intervención y de regulación sobre el mercado de trabajo y sobre las protecciones derivadas del trabajo.

De esta manera, la Sección I ofrece una mirada de largo plazo que intenta abarcar la evolución del mercado de trabajo argentino, haciendo especial foco en la distribución del ingreso. De dicha sección se desprende que los cambios políticos y económicos derivados del fin de la convertibilidad, por un lado, expresan y, por 
otro, generan diferencias sustanciales o estructurales. Así, la creación de puestos de trabajo se correspondió con niveles de desigualdad que apenas mejoraron. El caso argentino aparece ejemplificando la ambigua vinculación que se entabla entre el modo en que la riqueza es creada y su reparto, esto es, el modo en que capturan asalariados y no asalariados los resultados de la producción misma.

En tanto, la Sección II focaliza en los cambios y continuidades entre los años 90 y la etapa que sigue a la devaluación a través del análisis de los dos aspectos que hacen a la naturaleza del mercado de trabajo: el empleo y los ingresos. De los análisis se desprende que los cambios derivados de la devaluación (y de algunas medidas adoptadas en simultáneo) evidenciaron una fuerte y persistente recuperación del mercado de trabajo. Sin embargo, todavía no han podido alcanzarse los niveles de remuneraciones previos a la crisis, la mejoría en materia de precariedad laboral es aún insuficiente y persiste una fuerte heterogeneidad estructural reflejada en la segmentación del mercado de trabajo.

Finalmente la Sección III conecta de forma clara el mundo de la economía y el de la política a partir del análisis de intervenciones sobre el mercado de trabajo. Esas intervenciones se refieren tanto a los trabajadores actuales y a los que se retiraron, a las normativas específicas sobre el mundo de las relaciones laborales y a aquellas que lo trascienden.

De forma insoslayable la obra termina constituyendo un verdadero puntapié para el debate sobre uno de los temas centrales en nuestro país: la conformación o no de un régimen de acumulación, patrón de crecimiento o modelo económico diferente al vigente bajo el régimen de convertibilidad, y las potencialidades de esta nueva etapa para la construcción de una sociedad más inclusiva y más igualitaria.

\section{Bibliografía}

A. GRIMSON (2003), "La vida organizacional de zonas populares de Buenos Aires". Project "Latin American urbanization in the late $20^{\text {th }}$ century: a comparative study" - Center for the study of urbanization and internal migration in developing counties. Population Research Center, University of Texas at Austin. Working Paper Series 02. Project Second Meeting. Montevideo, Uruguay, agosto.

J. LINDENBOIM, Javier y C. DANANI (coord.) (2003), Entre el trabajo y la política. Las reformas de las políticas sociales argentinas en perspectiva comparada, Buenos Aires, Editora Biblos.

K. POLANYI (2006), La gran transformación, México, FCE (Edición original: 1944).

M-R. TROUILLOT (2001), "The anthropology of the state in the age of globalization. Close encounters of deceptive kind", en Current Anthropology, vol. 42, № 1. 